\title{
Magnetic cloud field intensities and solar wind velocities
}

\author{
W. D. Gonzalez, A. L. Clúa de Gonzalez, A. Dal Lago \\ Instituto Nacional de Pesquisas Espaciais, São José dos Campos, SP, Brasil \\ B. T. Tsurutani, J. K. Arballo, G. K. Lakhina, B. Buti, C. M. Ho \\ Space Plasma Physics, Jet. Propulsion Laboratory, California Institute of Technology. Pasadena,
}

S.-T. Wu

Center for Sipace Plasma and Aeronomic Research, and Department of Mechanical and Aerospace

Engineering, The University of Alabama in Huntsville, Huntsville

\begin{abstract}
.
For the sets of magnetic clouds studied in this work we have shown the existence of a relationship between their peak magnetic field strength and peak velocity values, with a clear tendency that clouds which move at higher speeds also possess higher core magnetic field strengths. This result suggests a possible intrinsic property of magnetic clouds and also implies a geophysical consequence. The relatively low field strengths at low velocities is presumably the cause of the lack of intense storms during low speed ejecta. There is also an indication that this type of behavior is peculiar for magnetic clouds, whereas other types of non cloud-driver gas events do not seem to show a similar relationship, at least for the data studied in this paper. We suggest that a field/speed relationship for magnetic clouds, as that obtained in our present study, could be associated with the cloud release and acceleration mechanism at the sun.

Since for magnetic clouds the total field tyically has a substantial southward component, $\mathbf{B}_{\mathbf{s}}$, our results imply that the interplanetary dawn-dusk electric field, given by $\mathbf{v} \times \mathbf{B}_{\mathbf{g}}$ (where $\mathbf{v}$ is the cloud's velocity), is enhanced by both factors. Therefore, the consequent magnetospheric energization (that is governed by this electric field) becomes more efficient for the occurrence of magnetic storms.
\end{abstract}

\section{Introduction}

It has been well established that major $\left(D_{s t} \leq-100\right.$ nT) magnetic storms are associated with fast Coronal Mass Ejections (CMEs) coming from the sun from both during solar maximum [Tsurutani et al., 1988, Goshng et al. 1991, Gonzalez et al., 1994] and the descending phase of the solar cycle [Tsurutani at al., 1995]. The energy transfer mechanism from the solar wind to the magnetosphere appears to be magnetic reconnection between the interplanetary magnetic field and the earth's magnetic field. 'The interplanetary dawn-dusk electric field, which is given by $\mathbf{v} \times \mathbf{B}_{\mathbf{s}}$, governs this process

\section{Copyright 1998 by the American Geophysical Union.}

Paper number 98GL00703.

0094-8534/98/98GL-00703\$05.00
[Dungey, 1961 ]. In the above expression, $\mathbf{v}$ is the solar wind velocity and $\mathbf{B}_{\mathbf{s}}$ is the southward component of the interplanetary magnetic field (IMF). Gonzalez and Tsurutani [1987] have established empirically that the interplanetary electric field must be greater than 5 $\mathrm{mV} / \mathrm{m}$ for longer than 3 hours to create a $D_{s t} \leq-100$ nT magnetic storm. This corresponds to a southward field component larger than $12.5 \mathrm{nT}$ for a solar wind speed of $\approx 400 \mathrm{~km} / \mathrm{s}$.

Although the positive correlation belween fast, ('MEs and magnetic storms have been stressed and is reasonably well understood, little attention has been paid to the opposite question, why don't slow CMEs lead to magnetic storms? Since this question involves the role of the magnetic field of the CME, it. leads to a more general question, namely how are the CME's speed and magnetic field related to each other? These are the questions we wish to address in this letter.

If the speed of the solar ejecta is less than the upstream slow solar wind plus the magnetosonic wave's phase velocity, a shock will not form at the leading (antisunward) edge of the ejecta, and there will not be compressed sheath fields. However, one might ask "why can't the ejecta themselves have fields intense enough to create magnetic storms with intensities $D_{s t} \leq-100$ nT?". Among the studied relationship of ejecta with magnetic storms, practically all intense storms were associated with magnetic clouds [ e.g. Burlaga et al., 1987; Tsurutani et al., 1988] that followed shocks, although Tsurutani ct al. [1988] claimed that the intense storm of September 18 1979, was associated with a magnetic cloud without a shock (with an average speed around $370 \mathrm{~km} / \mathrm{s}$ ). This latter type of association appears to be uncommon.

\section{Method of Data Analysis.}

To address this issue first we use magnetic cloud events. Magnetic clouds [e.g. Burlaga, 1995] are a type of ejecta that have a mesoscale dimension of about 0.25 $\mathrm{AU}$ at $1 \mathrm{AU}$ and crosses the spacecraft typically in about 24 hours. Magnetic clouds have a large and smooth rotation in the field's direction, an enhanced field strength and low proton temperature and $\beta$ values. It is thought [Farrugia et al., 1997 and references therein] that clouds 
are giant flux ropes formed by field aligned currents. The $B_{s}$ component of the cloud has typically an amplitude that represents a substantial fraction of the total IMF intensity. For example, for the intense and superintense storms studied by Tsurutani et al. [1988] and Tsurutani et al. [1992], the magnetic clouds responsible for about half of the storm events had their $B_{s}$ fields with intensities of $70 \%$ or more than that of the corresponding total IMF intensities. This association can explain why magnetic clouds with strong magnetic fields have typically strong $B_{s}$ components and therefore cause intense storms.

We have chosen to examine first previously published magnetic cloud events because their identification exists in the literature and the reader has the opportunity to examine the events in detail. We use (1) the five cloud events published and illustrated by Klein and Burlaga [1982]; (2) the two cloud events published and illustrated by Burlaga et al. [1987], using data from the IMP8/ISEE-3 satellites; (3) six cloud events reported by Tsurutani et al. [1988] and Tsurutani et al. [1992], using ISEE-3 data; (4) three cloud events discussed by Farrugia et al. [1997] who refer to previous studies that used data from the IM P8/ISEE-3 satellites; and (5) the one event reported by Burlaga et al. [1996], using data from the WIND satellite. All these 17 events were observed at $\approx 1 \mathrm{AU}$ and the criteria to select the events, that are common in all these cases, are: a large rotation in the ejecta field's direction, an enhanced field strength (typically $>10 \mathrm{n}^{\mathrm{r}} \mathrm{T}$ ), a low proton temperature and a duration of about 24 hours for the ejecta to cross the spacecraft.

Because most of these events were studied in association with intense magnetic storms, except the five events reported by $K l$ iein and Burlaga [1982], who selected clouds without any association with magnetic storms, we have looked for another (and independent) set of magnetic clouds that would not have an (a priori) association with magnetic storms. We obtained this second set of clouds in the following way. We took the whole year of 1979 , for which a full set of plasma and magnetic field data was recorded by the ISEE-3

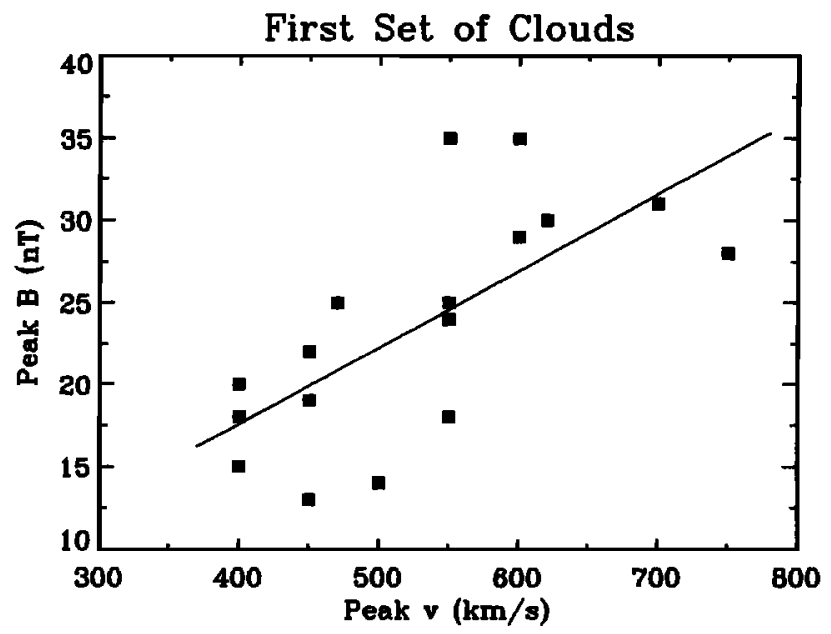

Figure 1. Scatter plot for $B_{\text {peak }}$ versus $v_{\text {peak }}$ for the first set of 17 magnetic clouds, taken from several references (see text), involving clouds that caused mostly intense and very intense magnetic stormıs.

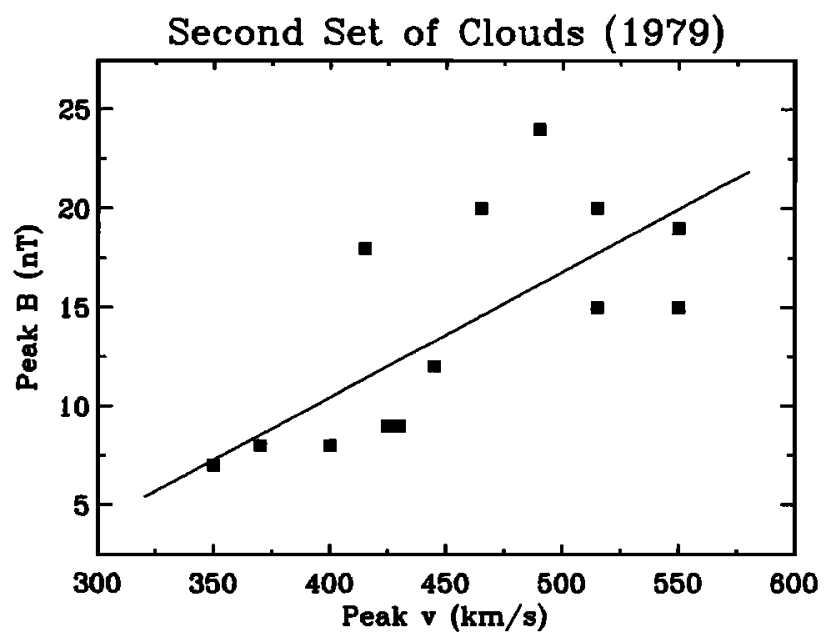

Figure 2. Scatter plot for $B_{p a k}$ versus vpak for the second set of 13 magnetic clouds, as observed by the ISEE-3 satellite in 1979. These events involved clouds that caused intense and moderate magnetic storms.

satellite, and identified the driver gas events using a combined criteria from those discussed by Zwickl et al. [1983] and Tsurutani et al. [1988]. The combined criteria involved: a smooth magnetic field (lack of Alfvén waves and discontinuities), high magnetic field strength, and a low proton temperature. For this study we have used 5 to 15 minute averages of the ISEE-3 data to identify the events and higher time resolution to check the smoothness of the field. These events were divided in two subsets, one including only clear magnetic clouds, using the selection criteria mentioned in the previous paragraph (with field rotation angles that for this data set lie in the domain of about 200 to 320 degrees), and the other subset was formed with the remaining driver gas-non cloud events. For the first subset we obtained 13 events and for the second one 24 events.

As for the first. set of clouds, we also performed the same study for the peak values of the solar wind speed and the magnetic field within the intervals of these two subsets of events.

It is interesting to point out that the second set of cloud events, even though they were selected without looking to their relationship to magnetic storms, they were later seen to still be associated with magnetic storms of at least moderate intensities (-100 nT $\leq D s t \leq-50 \mathrm{nT}$ ). One may think that this could be due to the fact that the year 1979 was near solar maximum, but it has been shown that the solar cycle distribution of storms does not necessarily follow the sunspot solar cycle distribution [e.g. Gonzalez et al., 1994].

On the other hand, it is also possible that magnetic clouds (following shocks) are most of the times associated with intense storms or at least with storms of moderate intensity, because they tend to involve fairly large values of $B_{3}$ [e.g. Burlaga ct al., 1987; Tsurutant et al., 1988].

\section{Results.}

The magnetic cloud intensity versus speed for each of the 17 events of the first set of clouds is shown in Figure 1 , in a scatter-plot format (Preliminary results related 


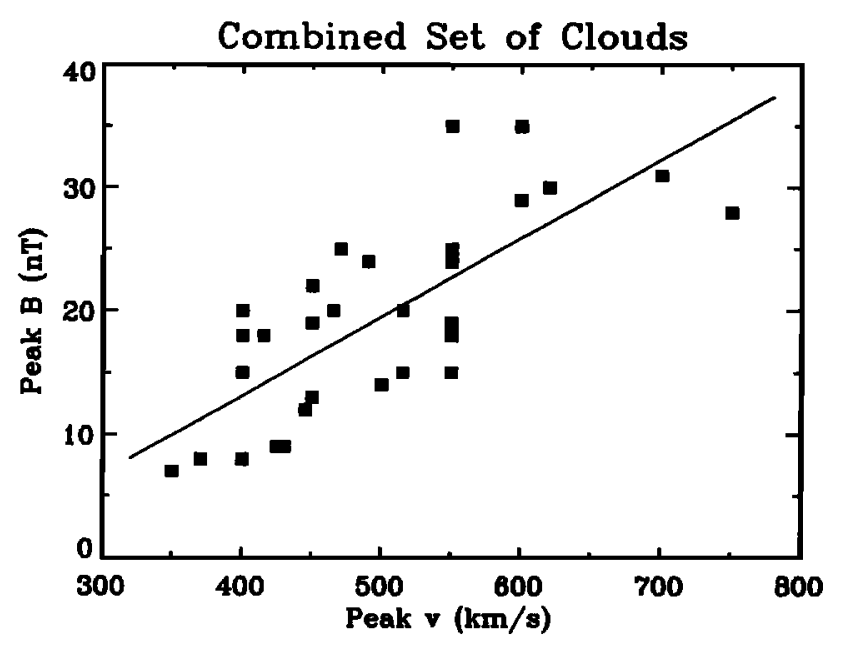

Figure 3. Scatter plot for the combination of the two independent sets of clouds shown in Figures 1 and 2.

to this figure were briefly mentioned by Tsurutani et al. [1998]). A linear regression fit is added to the plot. The correlation coefficient is 0.71 and the linear regression line gives,

$$
B_{\text {peak }}(n T)=0.047 v_{\text {peak }}(\mathrm{km} / \mathrm{s})-1.1
$$

Figure 2 shows a similar plot for the second set of clouds, namely for the 1979 driver gas-clear cloud events, and Figure 3 refers to the combined data set of those plotted in Figures 1 and 2, involving 30 clouds. The correlation coefficients obtained from the linear fits in Figures 2 and 3 are, respectively, 0.73 and 0.75 .

From Figure 3, we note that at cloud velocities of $v \approx 400 \mathrm{~km} / \mathrm{s}$, the field magnitudes can be 15 to $20 \mathrm{nT}$, which could have a $B$, component comparable to the value expected from the Gonzale $z$ and Tsurutani [1987] criteria, discussed in the Introduction. It is also interesting to note that the largely documented magnetic cloud event of Jan 10, 1997, with peak values of about. $15 \mathrm{nT}$ and $480 \mathrm{~km} / \mathrm{s}$, fits fairly well the trend shown in Figure 3.

Figure 4 refers to the ISEE-3 subset of driver gas-non cloud events. One can see that this plot is largely scattered, without any clear trend for a relationship between the peak values of the magnetic field and the solar wind speed. One can speculate that this set of events could still include clouds that were crossed by the satellite far from their center, for which a rotation in the field was much smaller. In such a case much lower $B$ fields are expected for a similar $v$ value of the cloud. It is possible that other driver gas structures could be involved in Figure 4, and deserve a detailed investigation.

\section{Discussion and Conclusions.}

For the sets of magnetic clouds studied in this work we have shown the existence of a relationship between their peak magnetic field strength and peak velocity values, that may suggest a possible intrinsic property of magnetic clouds and also imply a geophysical consequence. There is a clear tendency that clouds which move at higher speeds also possess higher core magnetic field strengths. The relatively low field strength at a low

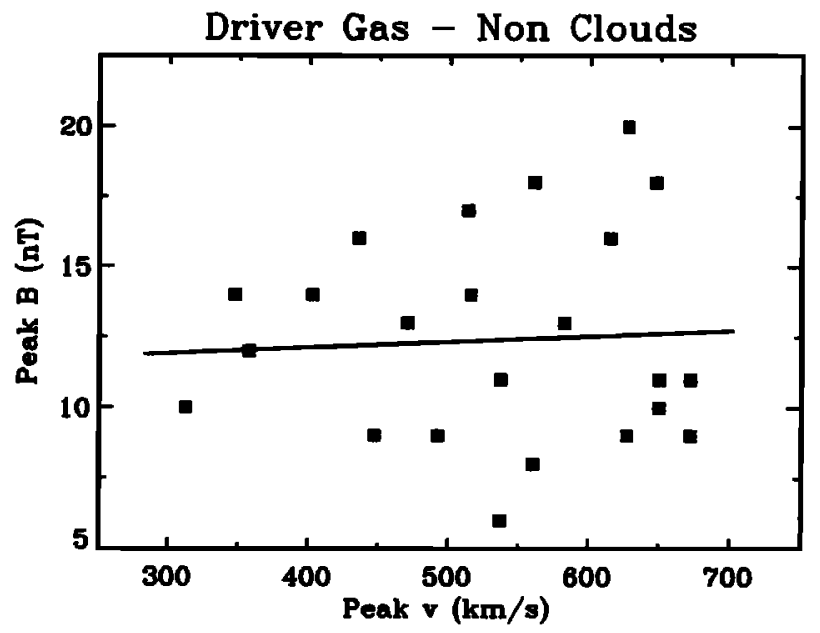

Figure 4. Driver gas events that apparently did not involve clouds in 1979 (see text), as measured by the ISEE-3 satellite.

velocity is presumably the cause of the lack of intense storms during low speed ejecta.

There is also an indication that this type of behavior is peculiar for magnetic clouds, whereas other types of non cloud-driver gas events do not seem to show a similar relationship.

Due to the $B-v$ relationship for magnetic clouds that we present in this paper, a faster cloud implies a larger amplitude of $B$ (which typically has a substantial $B$, component). Thus, since $B_{s}$ and $v$ are related to one another, the magnetospheric energy transfer, which is governed by $\mathbf{v} \times \mathbf{B}_{\mathbf{s}}$ [e.g. Gonzalez et al., 1994], is enhanced by both factors and therefore becomes more efficient for the occurrence of magnetic storms. This relationship excludes the possibility that a magnetic cloud with a high speed and a negligible or absent $B_{s}$ field could lead to the development of an intense storm. To our knowledge no one has observed such a case. This lack of observation also reinforces the $B-v$ relationship reported in our paper.

At this time, the physical causes of the reported relationship between the magnetic field and plasma speed of the magnetic clouds are uncertain. Compression of the cloud is certainly occurring. Thus, it is possible that in some cases the field increases can be accounted for by such an effect.

Another possibility is that this relationship may be associated with the CME release and acceleration mechanisms at the sun. For example, a recent self-consistent numerical MHD simulation study $[W u$ et al., 1997] shows that the radial velocity of the center of the flux rope (i.e. the center of the magnetic cloud), erupting from a helmet streamer, is proportional to the strength of the azimuthal component of the magnetic field, in a way consistent with the magnetic cloud $B-v$ relationship reported in this paper. A detailed parametric study of this kind of simulational work is currently under way.

Acknowledgments. Portions of this work were supported by the Fundo Nacional de Desenvolvimento Científico e Tecnológico of Brazil and by the National Aeronautics and Space Administration/ Jet Propulsion Laboratory, California Institute of Technology. 


\section{References}

Burlaga, L. F., Interplanetary Magnetohydrodynamics, Oxford University Press, New York, 1995.

Burlaga, L. F., K. W. Behannon, and L. W. Klein, Compound streams, magnetic clonds, and major geomagnetic storms, J. Geophys. Res., 92, 5725, 1987.

Burlaga, L. F., R. P. Lepping, W. Mish, K. W. Ogilvie, A. Szabo, A. J. Lazarus, and J. T. Steinberg, A magnetic cloud observed by Wind on October 18-20, 1995, NASA preprint, GSFC, February, 1996.

Dungey, J. W., Interplanetary magnetic field and the auroral zones, Phys. Rev. Lett., 6, 47, 1961.

Farrugia, C. J., L. F. Burlaga, and R. P. Lepping, Magnetic clouds and the quiet-storm effect at earth, in Magnetic Storms, edited by B. T. Tsurutani, W. D. Gonzalez, Y. Kamide and J. K. Arballo, Amer. Geophys. Union Press, Washington D. C., Geophys. Mon. Ser., 98, 91, 1997.

Gonzalez, W. D., and B.T. Tsurutani, ('riteria of interplanetary parameters causing intense magnetic storms (Dst < -100nT). Planetary Space Science, 35, 1101, 1987.

Gonzalez, W. D., J. A. Joselyn, Y. Kamide, H. W. Kroehl, G. Rostoker, B. T. Tsurutani, and V. M. Vasyliunas, What is a geomagnetic storm?", Review Paper. J. Geophys. Res., , 99 (A4), 5771, 1994.

Gosling, J. T., D. J. McComas, J. L. Phillips, and S. J. Bame, Geomagnetic activity associated with earth passage of interplanetary shock disturbances and coronal mass ejections. J. Geophys. Res., 96, 7831, 1991.

Klein, L. W., and L. F. Burlaga, Interplanetary magnetic. clouds at $1 \mathrm{AU}$, .J. Geophys. Res., 87, 613, 1982.

Tsurutani, B. T., W. D. Gonzalez, W. D., F. Tang, S. I. Akasofu, and E. J. Smith, Solar wind southward $B z$ features responsible for major magnetic storms of 1978-1979. J. Geophys. Res., 93 (A8), 8519, 1988.

Tsurutani, B. T, W. D. Gonzalez, W. D., F. Tang, and Y.
T. Lee , Great magnetic storms. Geophys. Res. Lett., 19, $73,1992$.

Tsurutani, B. T., W. D. Gonzalez, A. L. C. Gonzalez, F. Tang, J. K. Arballo, and M. Okada, Interplanetary origin of geomagnetic activity in the declining phase of the solar cycle. J. Geophys. Res., 100 (A11), 21717, 1995.

Tsurutani, B. T., J. K. Arballo, Y. Kamide, W. D. Gonzalez, and R. P. Lepping, Interplanetary causes of great and superintense magnetic storms, Physics and Chemistry of the Earth, in press, 1998.

Wu, S. T., W. P. Guo, and M. Dryer, Dynamical Evolution of a Coronal Streamer-Flux Rope System: II. A Self-Consistent Non-Planar Magnetohydrodynamic. Simulation, Solar Phys., 170, 265, 1997.

Zwickl, R. D., J. R. Asbridge, S. J. Bame, W. C. Feldman, J. T. Gosling, and E. J. Smith, Plasma properties of driver gas following interplanetary shocks observed by ISEE-3, in Solar Wind Five, NASA Conf. Publ., CP. 2280, 711 pp., 1983.

W. D. Gonzalez, A. L. Clúa de Gonzalez, A. dal Lago, Instituto de Pesquisas Espaciais, 12201-970, São José dos Campos, CP 515, SP, Brasil. (e-mail: gonzalez@dge.inpe.br; alicia@dge.inpe.br; dallago@dge.inpe.br)

B. T. Tsurutani, J. K. Arballo, G. K. Lakhina, B. Buti, C. M. Ho, Space Plasma Physics, Jet Propulsion Laboratory, California Institute of Technology, Pasadena, California, 91109. (e-mail: BTSURUTANI@jplsp2.jpl.nasa. gov; JARBALLO@ jplsp2.jpl.nasa.gov; LAKHINA@jplsp2. jpl.nasa.gov; BBUTI@jplsp2.jpl.nasa.gov; CHO@jplsp1.jpl. nasa.gov)

S.-T. Wu, Center for Space Plasma and Aeronomic Research, and Department of Mechanical and Aerospace Engineering, The University of Alabama in Huntsville, Huntsville, Alabama 35899. (e-mail: wus@cspar.uah.edu)

(Received November 10, 1997; revised December 26, 1997; accepted February 10, 1998.) 